\title{
Del escritorio a la tarima: una experiencia de formación en competencias transversales dentro de la licenciatura en biología
}

\author{
L. Rosich ${ }^{a}$, F. Bosch ${ }^{a, b}$, J.E. Baños ${ }^{b}$
}

\begin{abstract}
Introducción. Se diseñó una asignatura de pregrado con el fin de desarrollar competencias transversales en redacción científica y presentación oral. Materiales y métodos. Consistió en una asignatura de cuatro créditos en formato intensivo durante 2 semanas y al final se realizó una encuesta de evaluación de satisfacción, con una escala numérica (de 0 a 5, máxima satisfacción). Resultados. Los 20 alumnos dieron una puntuación de 4,4 a la asignatura en general, 4,5 a los contenidos teóricos y 4,8 a los prácticos sobre presentaciones orales. Consideraron que sus conocimientos y habilidades habían mejorado después de la asignatura. Conclusiones. El diseño de actividades dirigidas a la mejora de determinadas competencias transversales puede ser útil y es percibida satisfactoriamente por los estudiantes.
\end{abstract}

Palabras clave. Competencias genéricas. Comunicación oral. Redacción científica. Satisfacción de los estudiantes.

From the desk to the stand: a training experience in generic competences within the biology degree

Introduction. A new subject for undergraduates was designed in order to develop generic competences on scientific writing and oral communication. Materials and methods. It was organized as a 4 credit course in intensive format for 2 weeks. At the end of the course, an evaluation of the students' satisfaction was carried out by means of a numerical scale (from $0=$ worst to 5 very good). Results. All twenty students scored the course in general as 4.4,4.5 for lectures and 4.8 for the practical activities on oral presentations. Students felt that their abilities in scientific writing and oral communication have improved. Conclusions. The design of an education activity devoted to improve these cross-sectional competences could be useful and it is perceived positively by the students.
Key words. Generic competences. Oral communication. Scientific writing. Students'satisfaction.

\section{Introducción}

La correcta transmisión oral y escrita de los resultados de un estudio científico aún son tareas arduas para los profesionales de las áreas biomédicas. En muchas ocasiones, estas actividades se han considerado secundarias y han sido relegadas a un tipo de aprendizaje implícito en el trabajo diario, sin tratarse de forma específica en el contenido reglado de las diferentes carreras. Sin embargo, su asunción dentro del marco más amplio del Espacio Europeo de Educación Superior (EEES), concretamente dentro del proyecto Tuning, se considera en la actualidad un elemento importante de formación de los nuevos graduados [1]. Recientemente, se han realizado propuestas diversas que podrían ayudar a fomentar las competencias trasversales [2], pero se carece de descripciones empíricas de actividades específicas y de la valoración de los estudiantes sobre éstas.

La Universitat Pompeu Fabra (UPF) inició, en 1998, una licenciatura en Biología con una orientación biomédica clara. Su objetivo era formar biólogos con una preparación que permitiera su inserción en equipos multidisciplinarios de investigación y desarrollo de la biomedicina. Algunas actividades docentes de esta titulación han sido publicadas recientemente [3,4]. Desde el inicio de sus actividades, se tuvo una atención especial en el desarrollo de las competencias genéricas en los ámbitos de comunicación oral y escrita, trabajo en grupo y utilización de las tecnologías de la

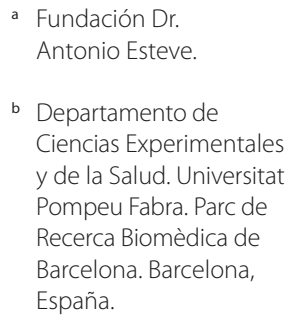

Correspondencia Dr. Fèlix Bosch.

Fundación Dr. Antonio

Esteve.

Llobet i Vall-Llosera, 2.

E-08032 Barcelona.

E-mail

fbosch@esteve.org

Agradecimientos Los autores quieren agradecer a los profesores y a los estudiantes que participaron en esta asignatura su entusiasmo y colaboración. La

financiación aportada por la Fundación Dr. Antonio Esteve contribuyó de forma importante a su realización. 
información. Previamente, se comunicó la experiencia de usar el portafolio para evaluar la asunción de tales competencias [5]. Sin embargo, no se ha descrito el empleo de actividades específicas destinadas a desarrollar algunas de ellas.

Con el objetivo de potenciar la formación en algunas competencias transversales de especial relevancia, la UPF, junto con la Fundación Dr. Antonio Esteve, decidieron impulsar la realización de una asignatura sobre redacción científica y presentaciones orales. El presente trabajo describe la experiencia y expone los resultados de esta iniciativa al valorar los contenidos, el formato y los conocimientos y habilidades adquiridos por parte de los estudiantes.

\section{Materiales y métodos}

\section{Descripción de la asignatura}

Durante el curso académico 2007-08 se ofreció una asignatura de créditos de libre elección sobre redacción científica y presentación oral, reconocida con cuatro créditos y dirigida especialmente a estudiantes de los últimos cursos de la licenciatura en Biología de la Facultad de Ciencias de la Salud y de la Vida de la UPF de Barcelona. Con el título 'Del escritorio a la tarima: redacción y presentación oral en biomedicina', la asignatura se organizó con carácter intensivo (5 horas diarias por las tardes) y se desarrolló durante 8 días de la segunda quincena de septiembre, cuatro de los cuales previos al inicio del período lectivo.

Su contenido, que se detalla en las tablas I y II, constó de dos bloques. El primero, dedicado a la redacción científica y al proceso editorial, ocupó un total de 20 horas, mientras que el segundo, que trató de la construcción y ejecución de las presentaciones orales, ocupó las 20 horas restantes. La asignatura se planteó con el doble propósito de dar a conocer las técnicas, estrategias y habilidades básicas para publicar y comunicar oralmente artículos científicos. Para conseguir dichos objetivos, se contó con la participación de 11 profesores procedentes tanto del ámbito académico, médico y científico, como del área de las artes escénicas. El número de plazas se limitó a una veintena de alumnos, con el fin de conferir a la asignatura una buena dinámica de participación con ejercicios prácticos, casos, debates y exposiciones orales.
Tabla I. Contenidos del apartado de redacción científica y proceso editorial.

- Aspectos generales sobre la comunicación y la divulgación científica. Importancia de la redacción científica y la presentación oral en el contexto de la comunicación biomédica. Introducción a la redacción científica: aspectos históricos, el estilo científico, revistas y formatos.

- Ética de las publicaciones. Las normas de Vancouver y la bibliografía. Datos fraudulentos o incompletos, vulneración de la confidencialidad, criterios de autoría, conflictos de intereses, publicación redundante y duplicada. Qué se debe referenciar y cómo hacerlo. Sistemas de citación y referencia.

- Ejemplos de redacción científica: el póster o cartel científico. Estructura y contenidos: título, introducción, material y métodos, resultados y discusión. Tablas, gráficos y figuras. Fondo y formatos. El informe o resumen técnico. Estructuración del informe y recomendaciones generales sobre los contenidos.

- El redactor científico. Una profesión y un punto de vista. Características del trabajo del redactor científico, perfil académico y ámbitos de trabajo.

- El artículo original. Definición y características generales. El título: la tarjeta de presentación del artículo. La firma bibliográfica. Estructura del artículo original: resumen, texto principal y otras secciones.

- El artículo de revisión. Revisión narrativa, revisión sistemática y metaanálisis. Estructura general de una revisión. Principales similitudes y diferencias entre el artículo original y el de revisión.

- Preparación y sumisión del manuscrito. Elección de la revista. El factor de impacto bibliográfico. La carta de presentación: más que una formalidad. Las comprobaciones finales del manuscrito. Los nuevos métodos de gestión de manuscritos.

- El complejo mundo del proceso editorial. Etapas del proceso editorial. Criterios de decisión. Resultados de la evaluación del manuscrito.

La evaluación final del rendimiento de los alumnos se realizó en función de tres aspectos básicos: la asistencia a las sesiones y la participación en los ejercicios conjuntos diferentes, la confección de un informe individual sobre ponencias diversas y, finalmente, mediante una presentación oral individual de 10 minutos de duración donde el alumno debía aplicar las técnicas aprendidas a lo largo del curso, con especial énfasis tanto en el 


\section{Tabla II. Contenidos del apartado de presentación oral.}

- Construcción de una presentación oral. Aspectos prácticos. El inicio: presentación y agradecimiento. Descripción de los datos científicos. ¿Cómo se presentan las tablas, gráficas y figuras? Las ayudas audiovisuales. Gráficos. Esquemas. Fondo y formatos. Efectos: animación, sonidos, transiciones. El final de la presentación.

- Planificación de la presentación oral. Conceptos generales de la comunicación oral. Elementos externos: audiencia, previsión y control del tiempo, auditorio, equipos. Diseño de los contenidos: título y presentación, introducción, cuerpo de la comunicación, conclusiones. Preguntas y comentarios finales.

- Claves de la expresión corporal. Autopresentaciones. Ejercicios de desbloqueo y activación de los sentidos a fin de facilitar la comprensión y práctica de los códigos de comunicación.

- Las técnicas de comunicación. Técnicas de respiración adaptación a la audiencia y relajación. Ejercicios de vocalización y ejercicios de proyección. Ejercicios corporales y de dicción. Aplicación de las técnicas anteriores.

- Ejecución y evaluación de las presentaciones. Ejercicios prácticos de integración entre las estrategias de construcción y ejecución de la presentación. Equilibrio entre la proyección y la presentación oral.

contenido científico como en los aspectos comunicativos, expresión verbal, modulación de la voz y lenguaje corporal, entre otros.

\section{Encuesta de evaluación}

Con el fin de conocer la opinión de los asistentes y poder ajustar los aspectos susceptibles de mejora de la asignatura, se solicitó a los alumnos que cumplimentaran una encuesta de evaluación a través de un formulario anónimo que se distribuyó el último día de clase. Se les pidió una valoración para cada uno de los aspectos solicitados mediante una puntuación de cada uno de ellos de 0 (pésima) a 5 (muy buena). Dicho cuestionario recogía la satisfacción de los alumnos, tanto sobre aspectos de los contenidos y formato del curso, como los conocimientos y habilidades adquiridos por los estudiantes, según su propia opinión. También se les ofreció la posibilidad de
Tabla III. Satisfacción de los alumnos con el curso ${ }^{a}$.

\begin{tabular}{|c|c|}
\hline & $\begin{array}{l}\text { Puntuación } \\
\text { media (DE) }\end{array}$ \\
\hline Valoración general de la asignatura & $4,4(0,5)$ \\
\hline $\begin{array}{l}\text { Contenido de las clases teóricas } \\
\text { sobre redacción }\end{array}$ & $3,8(0,6)$ \\
\hline $\begin{array}{l}\text { Contenido de las clases } \\
\text { sobre presentaciones orales }\end{array}$ & $4,5(0,6)$ \\
\hline $\begin{array}{l}\text { Valoración de los ejercicios } \\
\text { de redacción }\end{array}$ & $3,6(0,8)$ \\
\hline $\begin{array}{l}\text { Valoración de los ejercicios } \\
\text { de presentaciones orales }\end{array}$ & $4,8(0,5)$ \\
\hline Aportación del profesorado & $4,3(0,8)$ \\
\hline Horarios intensivos por la tarde & $3,7(0,7)$ \\
\hline Duración de la asignatura de 8 días & $4,3(0,9)$ \\
\hline $\begin{array}{l}\text { Realización en una semana } \\
\text { no lectiva y una lectiva }\end{array}$ & $4,2(1,0)$ \\
\hline $\begin{array}{l}\text { ¿Recomendarías esta asignatura } \\
\text { a tus compañeros? }\end{array}$ & $4,7(0,5)$ \\
\hline
\end{tabular}

a Puntuaciones según la escala entre 0 (valoración pésima) y 5 (valoración muy buena). DE: desviación estándar.

sugerir y destacar los aspectos que les habían resultado más y menos atractivos de la asignatura.

\section{Resultados}

Entre los resultados de la encuesta de evaluación (Tabla III) destaca, en primer lugar, la satisfacción de los estudiantes con la asignatura en su conjunto, valorada con una puntuación media de 4,4. De los parámetros evaluados, los alumnos 


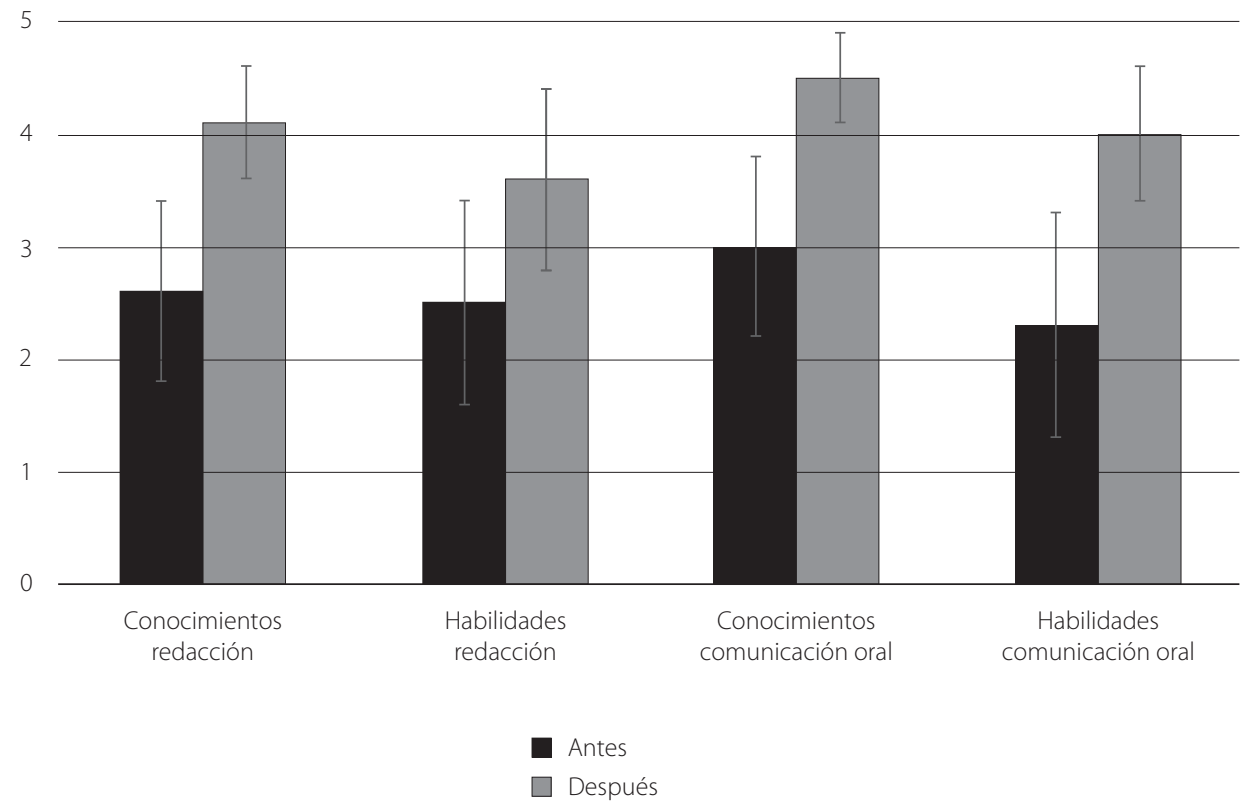

Figura. Puntuaciones medias (DE) de los conocimientos y habilidades antes y después de la asignatura según la apreciación de los estudiantes. Las puntuaciones de las ordenadas corresponden a una escala entre 0 (valoración pésima) y 5 (valoración muy buena).

coinciden en resaltar la aportación del profesorado $[4,3]$ y el programa dedicado a la construcción y ejecución de las presentaciones orales, tanto su contenido teórico $[4,5]$ como práctico $[4,8]$.

En la figura se representa la opinión de los estudiantes sobre la mejora de conocimientos y habilidades en redacción científica y presentación oral tras cursar la asignatura. Para ello, se preguntó la opinión sobre su situación anterior y posterior a la realización de este curso. En los cuatro ámbitos de estudio se observan mejoras, especialmente en las habilidades de comunicación oral (1,7 puntos de mejora media).

De las sugerencias recogidas mediante las preguntas abiertas de la encuesta, la mayoría de los alumnos destacó el contenido novedoso del curso y, en particular, las sesiones prácticas de expresión corporal y técnicas de comunicación. Asimismo, propusieron ampliar el contenido práctico del curso por su utilidad y aprovechamiento.

Finalmente, los alumnos reflejaron con contundencia $[4,7]$ su recomendación a compañeros para que realizaran el curso.

\section{Discusión}

Tradicionalmente, la formación en las disciplinas biomédicas se ha centrado en permitir que los estudiantes adquieran un cuerpo de conocimientos importante y habilidades prácticas destinadas a dominar las bases científicas de la profesión. Por esta razón, el tiempo destinado a dotarles de competencias transversales esenciales es muy reducido o, con frecuencia, inexistente. Esto a pesar de la evidencia de que tales competencias son especialmente estimadas en el ámbito laboral, por ejemplo, en farmacología [6], $\mathrm{y}$ de que parte de las actividades profesionales están destinadas a comunicarse con los colegas o a elaborar publicaciones que refrendarán el valor de su actividad científica. En reconocimiento de tal necesidad, el EEES recoge en sus principios la necesidad de que las competencias profesionales se consideren de forma explícita dentro de la formación académica del grado [7].

En los últimos años diversos estudios en el ámbito de la medicina $[8,9]$, la enfermería [10] o la 
psicología [11] han reforzado la importancia de diseñar actividades específicas para formar de forma más adecuada a los estudiantes de estas disciplinas. Generalmente, las actividades que contemplan de forma sincrónica la comunicación oral y escrita son más escasas y los ejemplos se limitan, en general, al ámbito de la formación posgraduada [12]. 'Del escritorio a la tarima' ha sido bien acogida por los estudiantes que han evaluado muy positivamente su realización. Parece deseable que los nuevos planes de estudio incluyan actividades comunes destinadas a desarrollar de forma explícita estas actividades y los créditos asignados a la rama de conocimiento en que se adscribe el título pueden ser un buen lugar para ofrecerlos.

\section{Bibliografía}

1. González J, Wagenaar R. Tuning education structures in Europe. Pilot Project-Phase 3. URL: http://tuning. unideusto.org. [15.12.2007].

2. Baños JE, Pérez J. La importancia de las competencias genéricas en los estudiantes de ciencias de la salud: algunas propuestas de aplicación. Educación Médica 2005; 8: 216-24.

3. Baños JE, Aramburu J, Sentí M. Biocinema: the experience of using popular movies in the teaching of biology. J Med Mov 2005; 1: 42-6.
4. Aramburu J, Bosch F, Sentí M, Baños JE. Los itinerarios profesionales como método para mejorar la inserción profesional de los licenciados en biología: la experiencia de la Universitat Pompeu Fabra. Educación Médica 2006; 9: 23-30.

5. Valero M, Aramburu J, Baños JE, Girvent M, Pérez J, Sentí M. Introducción del portafolio: dificultades y satisfacción. Educación Médica 2005; 8: 155.

6. Hughes I, Hollingsworth M, Jones SJ. Knowledge and skills needs of pharmacology graduates in first employment: how do pharmacology courses measure up? TiPS 1997; 18: 111-6.

7. Towards the European Higher Education Area: responding to challenges in a globalised world. Conference of European Higher Education Ministers. Contribution of the European Commission. Londres, 17-18 de mayo. URL: http://www.bmwf.gv.at/fileadmin/user_upload/ europa/bologna/eu_comm_minconf.pdf. [18.12.2007].

8. Marusic A, Marusic M. Teaching students how to read and write science: a mandatory course on scientific research and communication in medicine. Acad Med 2003; 78: 1235-9.

9. Rawson RE, Quinlan KM, Cooper BJ, Fewtrell C, Matlow JR. Writing skills developments in the health professions. Teach Learn Med 2005; 17: 233-9.

10. Whitehead D. The academia writing experiences of a group of student nurses: a phenomenological study. J Adv Nursing 2002; 38: 498-606.

11. Fallahi CR, Word RM, Austad CS, Fallahi H. A program for improving undergraduate psychology students' basic writing skills. Teaching Psychol 2006; 33: 171-5.

12. Surratt CK. Creation of a graduate oral/written communication skills course. Am J Pharm Educ 2006; 70: 1-8. 
